\title{
A Developmental Framework of Brain and Cognition from Infancy to Old Age
}

\author{
Alvaro Pascual-Leone $\cdot$ Margot J. Taylor
}

Received: 28 July 2011 / Accepted: 28 July 2011/Published online: 20 August 2011

(C) Springer Science+Business Media, LLC 2011

The interest in lifespan studies has grown tremendously in recent years with awareness of the possible parallels between development and ageing processes, and also with the increasing knowledge of the malleability of the brain even into old age and the continuing opportunities for ongoing learning and interventions across the lifetime. Our conceptualization of brain changes and brain function across the lifespan has evolved from the vision of two distinct and separate processes, an initial development till maturity, and a later decline through ageing processes (Fig. 1a), to a much more dynamic, life-long vision in which processes evolve and influence each other across the entire lifespan (Fig. 1b). There is growing awareness in the neurosciences of the importance of understanding brain development from infancy (and even in utero) through childhood and then the continuing evolution of brain structure and function in adult and then ageing populations. To fully appreciate a mature or ageing system, it is important to understand the processes underlying and contributing to its maturation, and how related processes continue to shape brain structure and function until death. We have developed the appreciation that there is no period when the brain and its functions are static; changes are

This is the Introduction to the Special Issue: Brain function across the lifespan.

\section{A. Pascual-Leone}

Berenson-Allen Center for Noninvasive Brain Stimulation,

Cognitive Neurology Unit and Department of Neurology,

Beth Israel Deaconess Medical Center, Harvard Medical School,

Boston, USA

M. J. Taylor $(\square)$

Diagnostic Imaging, Hospital for Sick Children,

University of Toronto, Toronto, Canada

e-mail: margot.taylor@sickkids.ca continuous throughout the lifespan. The nature, extent and the rates of change vary by region and function assessed and are influenced by genetic and well as environmental factors, both due to physiologic influences and by diseasesrelated pathological processes.

This special issue offers an array of papers, which incorporate literature reviews for those not as familiar with the field of brain lifespan studies, with their empirical data. The studies included are exceptional in their application of complex protocols that assess the neuroplasticity and agerelated changes in structure and/or function from infants through to our most senior citizens, and highlight the value of different methodological techniques and approaches.

Starting this special issue are two letters addressing the topic of measures of brain maturity and providing commentary on Dosenbach et al. (2010). Brandeis et al. (2011) raise the point that estimation of brain maturity has a long history, as it has been assessed for many decades using electrophysiological measures. Dosenbach et al. (2010) agree with the suggestion that neuroimaging approaches be combined. These letters raise the intriguing questions of commonalities across imaging modalities that should be kept in mind for all of the papers in this special issue.

We have organized the reports from early to late in the lifespan. The topic of the first paper is on the youngest of participants-4-month-old infants. Trainor and colleagues (2011) presented young infants with one of two types of musical stimuli over the course of a week. After only about $160 \mathrm{~min}$ of listening experience, event-related potential (ERP) responses in the infants from the two different groups reflected the musical exposure of the infant. This very interesting paper demonstrates the early and rapid plasticity in the infant brain. The following study by Gordon and colleagues (2011) also investigated the neuroplasticity of brain function; in this case in infants and 


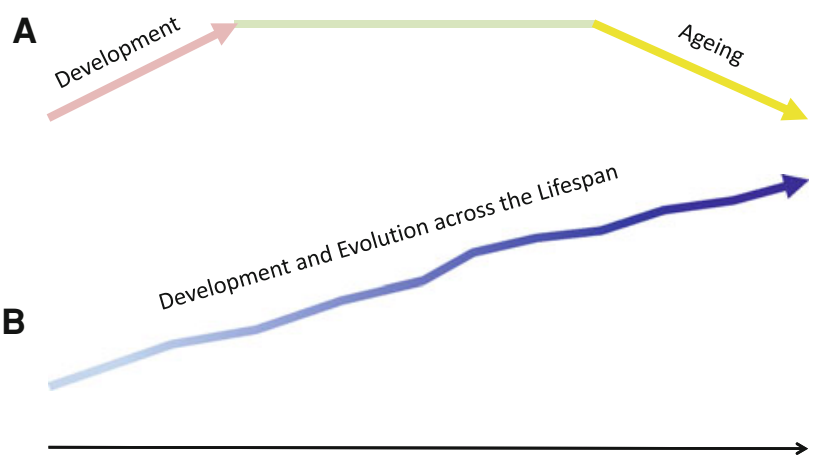

Age [years]

Fig. 1 Schematic representations of two views of brain and cognition changes across the lifespan. Curve A shows the traditional view of lifespan studies; there is a developmental process followed by a stable adult period, followed by decline in ageing. With this special issue, we hope to encourage colleagues to view brain function across the lifespan as a continuing, modulating and evolving process (curve $B$ )

children who are deaf and received cochlear implants. This paper first provides an excellent review of the issues related to the effects of impaired sensory input and how that impacts the development of the auditory system. The data presented are extensive in showing that the timing of the implant can affect different levels of plasticity within the auditory system, with less plasticity being seen in the cortical regions in fact. This paper is also valuable in its integration of information from human and animal studies. In a third study that involved infants, Luyster et al. (2011) studied 12 month olds who were at high risk for ASD compared to low risk infants. As social interactions are often one of the first indications of ASD, they studied ERPs to familiar and unfamiliar faces. At this early age there were as yet no striking differences between the two groups, although there was some evidence for a lag in the high risk group. This paper discusses in detail the challenges of early identification of infant ASD and the definition of the ASD endophenotype at a very young age.

The next few studies are focused on childhood. The first of these, by Pannekamp et al. (2011), investigated language discourse, examining the complex issue of context and prosody in children from early childhood to pre-adolescence. The language used in the task was German where prosodic accents are very critical; only the 12 year olds had ERPs that reflected the contextual information independent of prosody, as seen in adults. The ERPs of the younger children ( 8 and 5 year-old age groups) demonstrated their successful processing of sentences, but they were more influenced by the dominant prosodic information in the dialogue. In contrast, the paper by Qui et al. (2011) assessed brain structure and function relations in the absence of a task, examining cortical thickness and DTI measures in relation to resting state functional networks in children with and without ADHD. The convergence among structural and functional measures holds great promise for advancing our understanding of typical and atypical brain development, as shown in these preliminary data.

The following three papers use the neuroimaging modality of magnetoencephalography (MEG). In the first of these, Pang (2011) reviews the practical aspects of developmental studies using MEG. This is an invaluable addition to the literature, given the increasing use of MEG in developmental studies including atypical populations. Comparable papers have been published for other imaging modalities, in particular fMRI, but the approaches and issues are different with MEG; thus this is an important guide and we are sure will be a widely used reference paper. In the following paper by Taylor et al. (2011) use MEG to assess face recognition from young children through to adults, with a focus on hippocampal and frontal lobe activations. They show that right hippocampal activity was visible from 6 years of age, but frontal lobe activations emerged only in mid-childhood. These data demonstrate the parallels between structural and functional development that extend into adulthood. The following paper by Herdman and colleagues (2011) also looks at development in children compared to adults, in the auditory modality. These authors investigated attentional effects to pure tone stimuli and found that the modification of the MEG attentional effects was limited in the 12 year olds. They suggest that these findings support the model that with experience attentional gating occurs at increasingly early levels in the auditory system to facilitate automatic processing.

The next pair of papers relates to age-related changes in normal adults and ageing. McGinnis and colleagues (2011) focus on cortical thickness changes with ageing. They test the popular "last in, first out" hypothesis. They demonstrate that the cortical thinning does not follow a linear pattern and that the changes between young and middleaged adults are greatest in different areas than those found between the middle and older-aged adults. They propose a new model of cortical changes with age and highlight how this model will allow for interventions that are specific to the age-related changes over the adult lifespan. The following paper by Falvo and colleagues (2011) investigates the cortical activation related to motor movements in young and old adults. While peripheral measures did not differ as a function of age, the cortical activation of three ERP measures related to movement preparation and performance did, and suggests that there is less focal activation in older adults during motor movements. This paper concludes that the adaptable nature of cortical networks governing motor function may be attenuated in older adults, requiring support from additional brain regions.

The final four papers in this special issue compare healthy and pathological ageing processes. The first by 
Pascual-Leone et al. (2011) brings multi-modal imaging techniques-TMS, EEG and fMRI-to bear on questions of neuroplasticity and how this is affected by age and by various disease states. This paper includes an extensive review of the literature and proposes an integrative model. The second paper by Foy and colleagues (2011) examines magnetic resonance spectroscopy to determine if there are neurometabolic biomarkers to identify the early stages of Alzheimer's disease (AD). The aim is to identify such subjects as soon as possible to allow early interventionsan important clinical issue in ageing. This study investigated patients with $\mathrm{AD}$ compared to people with mild cognitive impairment and healthy age-matched controls and found that some combinations of the MRS data could distinguish amongst the groups. To further explore the effects of ageing on memory and other cognitive function, Aine et al. (2011) review of a series of their studies that examined several of the critical issues related to the reports of age-related memory decline in the elderly. They propose a number of limitations that need to be considered in the interpretation of these data in the literature and that are important for accurate ongoing and future studies. The final paper in this issue also deals with the age-related changes and the models of capacity in the ageing brain. Bartres-Faz and Arenaza-Urquijo (2011) focus on the structural capacity of the ageing brain compared to the cognitive capacity or reserve of the brain. As with the preceding three papers, this review also highlights the responsibility of understanding brain structure in light of brain function, with an emphasis on the interactions among aspects of mental activity, the neuroprotection and compensatory mechanisms. A final point that is raised in this paper, as well as in some of the other papers in this special issue, is the importance of longitudinal studies.

Most of the work included in this special issue of Brain function across the life span does not include longitudinal studies, which are extremely difficult to run and to manage over the many years that are required to obtain those data. However, a number of groups internationally are increasingly including longitudinal aspects in their investigations of development of the brain over the lifespan, and this will be an important direction for the future.

This special issue highlights the scope of neuroscience research across the lifespan. The studies used protocols and paradigms with ethological validity and emphasize the range of neuroimaging measures that can be utilized in the examination of brain structure and function over the lifetime. Infant studies are fundamental and mandatory for our understanding of innate capacities and initial factors that influence early maturation; we are grateful for the exceptional work of our colleagues in this time-intensive and demanding field. Studies with children demonstrate that brain processing, even for simple stimuli and certainly for more complex stimuli such as language, is far from mature in childhood; there is extensive cognitive development continuing throughout the adolescent years (despite what our teenagers tell us). Studies in adults moving through middle age and into old age are critical to understand the underlying causes for decline in various functions with age; this knowledge will contribute to interventions that will slow the onset or the progression of these age-related changes. The clinical studies complement normative studies by providing atypical cognitive profiles, which help define our models of human information processing.

We would like to thank the editors, Dr. Micah Murray and Dr. Christoph Michel for the privilege of editing this Special Issue on Brain function across the lifespan, and all the authors for their contributions. We are grateful for our many reviewers for their careful assessments of the manuscripts, constructive and insightful suggestions, as well as their willingness to review in a very timely manner. We would also like to thank Jared Horvath for his invaluable administrative assistance. We hope that this focused special issue will be of interest to a wide range of researchers in the field of cognitive neurosciences, neurology and paediatric neurology, psychiatry, geriatrics and hope that it will help spur further lifespan studies.

\section{References}

Aine CJ, Sanfratello L, Adair JC, Knoefel JE, Caprihan A, Stephen JM (2011) Development and decline of memory functions in normal, pathological and healthy successful aging. Brain Topogr. doi: 10.1007/s10548-011-0178-x

Bartrés-Faz D, Arenaza-Urquijo EM (2011) Structural and functional imaging correlates of cognitive and brain reserve hypotheses in healthy and pathological aging. Brain Topogr (this issue)

Brandeis D, Koenig T, Wackermann J (2011) Individual brain maturity: from electrophysiology to fMRI (Letter). Brain Topogr. doi: 10.1007/s10548-011-0184-Z

Dosenbach NUF, Petersen SE, Schlaggar BL (2011) Individual brain maturity: from electrophysiology to fMRI-response (Letter). Brain Topogr. doi: 10.1007/s10548-011-0185-y

Falvo MJ, Sirevaag EJ, Rohrbaugh JW, Earhart GM (2011) Central adaptations to repetitive grasping in healthy aging. Brain Topogr. doi: 10.1007/s10548-011-0183-0

Foy CML, Daly EM, Glover A, O'Gorman R, Simmons A, Murphy DGM, Lovestone S (2011) Hippocampal proton MR spectroscopy in early Alzheimer's disease and mild cognitive impairment. Brain Topogr. doi: 10.1007/s10548-011-0170-5

Gordon KA, Wong DDE, Valero J, Jewell S, Yoo P, Papsin BC (2011) Use it or lose it? lessons learned from the developing brains of children who are deaf and use cochlear implants to hear. Brain Topogr. doi: 10.1007/s10548-011-0181-2

Herdman AT (2011) Neuroimaging evidence for top-down maturation of selective auditory attention. Brain Topogr. doi: 10.1007/ s10548-011-0182-1

Luyster RJ, Wagner JB, Vogel-Farley V, Tager-Flusberg H, Nelson CA (2011) Neural correlates of familiar and unfamiliar face processing in infants at risk for autism spectrum disorders. Brain Topogr. doi: 10.1007/s10548-011-0176-Z 
McGinnis SM, Brickhouse M, Pascual B, Dickerson BC (2011) Agerelated changes in the thickness of cortical zones in humans. Brain Topogr. doi:10.1007/s10548-011-0198-6

Pang EW (2011) Practical aspects of running developmental studies in the MEG. Brain Topogr. doi: 10.1007/s10548-011-0175-0

Pannekamp A, van der Meer E, Toepel U (2011) Context- and prosody-driven ERP markers for dialog focus perception in children. Brain Topogr. doi: 10.1007/s10548-011-0194-x

Pascual-Leone A, Freitas C, Oberman L, Horvath JC, Halko M, Eldaief M, Bashir S, Vernet M, Shafi M, Westover B, Vahabzadeh-Hagh AM, Rotenberg A (2011) Characterizing brain cortical plasticity and network dynamics across the agespan in health and disease with TMS-EEG and TMS-fMRI. Brain Topogr (this issue)
Qiu M, Ye Z, Li Q, Liu G, Xie B, Wang J (2011) Changes of brain structure and function in ADHD children. Brain Topogr. doi: 10.1007/s10548-010-0168-4

Taylor MJ, Mills T, Pang EW (2011) The development of face recognition; hippocampal and frontal lobe contributions determined with MEG. Brain Topogr. doi: 10.1007/s10548-0110192-Z

Trainor LJ, Lee K, Bosnyak DJ (2011) Cortical plasticity in 4-monthold infants: specific effects of experience with musical timbres. Brain Topogr. doi: 10.1007/s10548-011-0177-y 